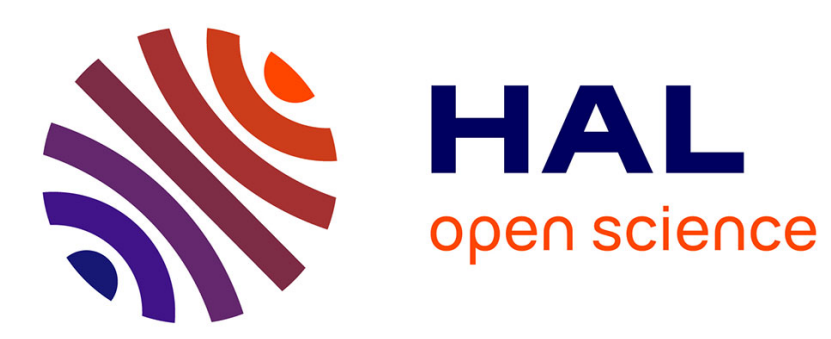

\title{
Duration-aware Data Collection in UAV-aided Mobile Sensor Networks
}

\author{
Xiaoyan Ma, Tianyi Liu, Rahim Kacimi, Riadh Dhaou, Song Liu
}

\section{To cite this version:}

Xiaoyan Ma, Tianyi Liu, Rahim Kacimi, Riadh Dhaou, Song Liu. Duration-aware Data Collection in UAV-aided Mobile Sensor Networks. International Wireless Communications and Mobile Computing Conference (IWCMC 2021), IEEE Communications Society, Jun 2021, Harbin (Virtually), China. hal-03185684

\section{HAL Id: hal-03185684 https://hal.science/hal-03185684}

Submitted on 30 Mar 2021

HAL is a multi-disciplinary open access archive for the deposit and dissemination of scientific research documents, whether they are published or not. The documents may come from teaching and research institutions in France or abroad, or from public or private research centers.
L'archive ouverte pluridisciplinaire HAL, est destinée au dépôt et à la diffusion de documents scientifiques de niveau recherche, publiés ou non, émanant des établissements d'enseignement et de recherche français ou étrangers, des laboratoires publics ou privés. 


\title{
Duration-aware Data Collection in UAV-aided Mobile Sensor Networks
}

\author{
Xiaoyan Ma ${ }^{1}$, Tianyi Liu ${ }^{2}$, Rahim Kacimi ${ }^{3}$, Riadh Dhaou ${ }^{4}$ and Song Liu ${ }^{1}$ \\ ${ }^{1}$ College of Architecture and Urban Planning, Tongji University, Shanghai, China; \{xiaoyan_ma, liusong5\}@tongji.edu.cn \\ ${ }^{2}$ School of Aerospace Engineering and Applied Mechanics, Tongji University, Shanghai, China; tianyi.liu@tongji.edu.cn \\ 3 IRIT-UPS, Toulouse University, Toulouse, France; kacimi@irit.fr \\ ${ }^{4}$ IRIT-ENSEEIHT, Toulouse University, Toulouse, France; riadh.dhaou@ toulouse-inp.fr
}

\begin{abstract}
Unmanned Aerial Vehicle (UAV)-aided Wireless Sensor Network (WSN) has been received increasing attentions in recent years due to its tremendous applications in many fields. The high dynamic topology in such network brings new communication challenges. In this paper, we address on the dynamic parameters, including the velocity and flying height of the UAV, the sensors velocities, and its real-time location, which affect the topology and influence the communication performance of the system. We introduce the contact-duration to mathematically integrate these parameters and to measure the transmission opportunity between the mobile node and the UAV and formulate the data collection issue as an optimization problem with the objectives of jointly maximizing the number of collected packets and the number of nodes that participate the communications with the UAV. Furthermore, we propose an opportunity-optimal frame selection algorithm, named as OFS algorithm, to increase the communication opportunities of the nodes, thereby, the data collection performance of the network was enhanced. Through extensive simulations based on simulated movement and real experiments, we evaluate the effectiveness of the proposed mechanisms under different configurations. The results present that the proposed mechanisms perform well on enhancing the transmission opportunity of the mobile nodes and the data collection performance of the network.

Index Terms-Wireless sensor networks, unmanned aerial vehicle, mobility, opportunistic communications, contact duration prediction
\end{abstract}

\section{INTRODUCTION}

Unmanned aerial vehicles (UAV) aided wireless sensor network (WSN) have achieved tremendous applications ranging from agriculture, industry, healthcare, to military [1]. In applications, one of the main roles of the UAV is used as a mobile collector [2]. Many researches have been done on data collection issues in the context of UAV-aided WSN [1]-[3]. Most of these researches address on deterministic topology where the sensor is static deployed its positions could be known through different methods. The data collection issues which studied on dynamic topology are seldom covered [4].

The main difference between the static deployment and mobile setting in UAV-aided WSN is: the topology in mobile case is much high dynamic than that in static case. In static network, all sensors are static deployed, and only the UAV is mobile. The flying information of the UAV including the trajectory could be designed in advanced and be well controlled through professional flight control system [4]. However, in mobile case, the movements of the targets are unknown

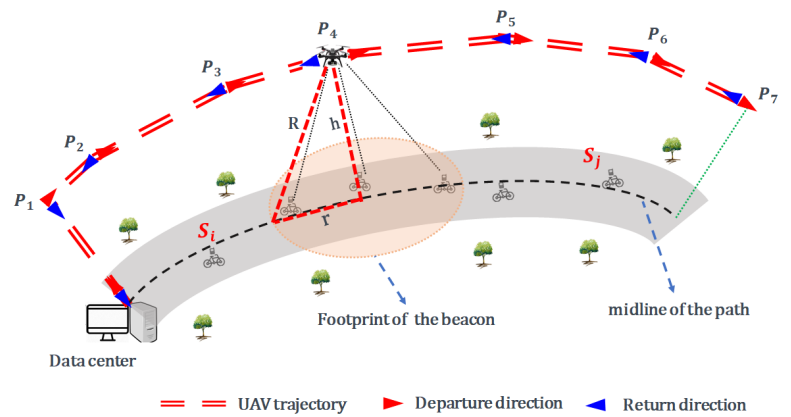

Fig. 1. Studied scenario.

and it could be randomly in some applications such as animal tracking etc. It brings new challenges to the traditional data collection algorithms because that the target already out of the range of the collector when it is the target's turn to send data.

In such applications, the key point is the duration of a sensor when it is within the range of the UAV, which is named as contact duration between the sensor and the UAV in this paper. Intuitively, a sensor has high opportunity to send data to the UAV when it has long contact duration with the UAV. On the contrary, a sensor has small opportunity to send data if it has short contact duration with the UAV. In the existing data collection algorithms, these kind of sensors may lost the opportunities to send data if the contact duration is not considered in the algorithms.

To the best of our knowledge, the existing work mainly focus on deterministic cases, where the movement of the sensor and the UAV are known in advance or could be precise controlled [5], [6]. In such research, the contact duration between the sensor and the UAV could be accurately calculated. This is not applicable for mobile sensor network because of the changes in sensor velocity are difficult to known in advance. The change of velocity includes two aspects, the change of direction and the change of speed. Generally, neither of these changes can be known in advance in applications. The key point is to predict the movement of the sensor within a period in the future through the movement state that has occurred in the past. Correspondingly, the prediction of contact duration is obtained.

In this work, we focus on how to predict the contact duration 
precisely so as to enhance the transmission opportunities of those nodes that have short contact duration, thereby, enhance the data collection performance of the network. Fig. 1 illustrates an application scenario in the context of UAV-aided mobile sensor network. The data collection maximization contains: $(i)$ the maximizing of collected packets, and (ii) the maximizing of the number of nodes that participate the communications. In this work, our main purpose is to jointly maximizing the two objectives. The main contributions in this paper are:

- We study the impact of dynamic parameters in UAV-aided mobile WSN, and formulate the data collection issue into an optimization problem with the objectives of jointly maximizing the number of collected packets and the number of sensors that participate the communications.

- We propose a mechanism which is applicable to predict the contact duration precisely through mathematically integrating the dynamic parameters.

- We propose a opportunity-optimal frame selection algorithm, named as OFS algorithm, to conduct the data collection between the mobile node and the UAV. It enhances the transmission opportunities of those nodes that have short contact duration with the UAV. Thereby, it enhances the number of nodes which participated the data collection.

- Through extensive simulations based on simulated movement and real experiments, we compare the predicted duration with real value, and examine the effectiveness of proposed data collection mechanism under different configurations.

This paper is organized as follows: the next section presents the related work. In Section III, we introduce the system model and propose solutions in Section IV. We evaluate the proposed algorithms through extensive simulations based on simulated movement and real experiments in section V. The section VI concludes this work and gives some future work suggestions.

\section{RELATED WORK}

Numerous research have been done on data collection in UAV-aided WSN. In this section, these algorithms are differentiated by whether the sensor is mobile or not. This is because that the dynamic parameters brought by the sensor movement have significant impact on the system performance of data collection. In the following, we classify these data collection algorithms by the criteria: static or mobile nodes.

Tremendous research with different objectives have been done on UAV-aided static sensor network. In [7]-[10], the authors use UAV to collect data from static nodes through planning the UAV trajectory with the objective of minimizing the system energy consumption [7], the delay [8], and the average data collection rate [9], and also of maximizing the received energy maximization of users [10]. Other works focus on the jointly optimization of the trajectory, the height, the speed, etc. For example, to minimize the total collecting time of the UAV through jointly optimizing the UAV trajectory, fly height and velocity, and data links with ground users [11].

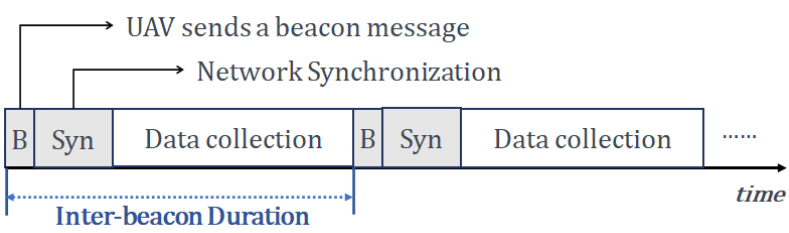

Fig. 2. The procedure of the studied scenario. The width of the rectangle in this figure is not proportional to the duration of sending this message.

In [8], they aim to minimize the maximum delay of all users through jointly optimizing the UAV trajectory, the scheduling variables of the users, and the offloading ratio. And in [2], the authors aim to maximize the number of served devices through jointly optimizing the UAV trajectory and the allocation of the radio because of the limited buffer size of the device and the timeliness of their reading.

Seldom research focus on mobile sensor network. In [5], [6], [12], the authors use a UAV to collect data from mobile nodes through jointly optimizing the velocity and the flying height of the UAV. However, these researches are done on an assumption that the trajectories of the nodes and the UAV are straight lines and both of them move with constant velocities. Under such assumption, the movements for the UAV and the sensors are precisely calculated. The generally mobile case are not considered.

Although many works have been studied, there still a room for enhancing the system performance through jointly considering the dynamic parameters.

\section{Network Model and Problem Formulation}

\section{A. Network Model}

We study a UAV-enabled mobile sensor network which consists of a UAV and multiple sensors as shown in Fig. 1. The data center is set at the original center point of the path. Sensors are deployed in the front part of the predefined path and move along the path. The UAV takes-off from the data center and fly to the given height $h$, and then, fly along the path with a given speed $v$ to collect data from sensors.

From Fig. 1, the UAV trajectory consists of a few number of line segments which contain several waypoints. The UAV's collecting time is the duration when the UAV flying from $P_{1}$ to $P_{E}$ with a given speed $v$ as shown in Fig. 1 .

It can be noticed from Fig. 1 that both mobile of the UAV and the nodes makes the network having high dynamic topology. To make it proper functioning, we use periodic beacon mechanism to synchronize the network. The UAV sends a beacon message to its coverage to tell it is coming (as shown in Fig. 2). The sensors that received the beacon send join messages which contain the locations and speeds to the UAV. After the reception of the join messages from nodes, it starts the data collection. The duration between adjacent two beacon is named as inter-beacon duration (IBD).

It is worth notice that different sensors have different contact duration with the UAV because of different speeds and 
locations which means that they have different transmission opportunities with the UAV. The key challenge is: how to balance the transmission opportunities between them so as to enhance the data collection of the network.

\section{B. Channel Model}

To well implement the air-ground communication, both lineof-sight (LoS) and non-line-of-sight (NLoS) links [13] are considered in this paper. The LoS probability in $t_{k}$ is given by [15],

$$
P_{L, S_{t_{k}}^{i}}=\frac{1}{1+a \cdot \exp \left[-b\left(\frac{180}{\pi} \cdot \arcsin \left(\frac{h}{d_{t_{k}}\left(U, S_{i}\right)}\right)-a\right)\right]},
$$

where $a$ and $b$ are constants, and $d_{t_{k}}\left(U, S_{i}\right)$ is the distance between sensor $S_{i}$ and the UAV at $t_{k}$. Then, the NLoS probability is,

$$
P_{N L, S_{t_{k}}^{i}}=1-P_{L, S_{t_{k}}^{i}},
$$

Then, the path loss is given by,

$$
L_{S_{t_{k}}^{i}}=l_{\sigma} \cdot\left(\frac{4 \pi \cdot f \cdot d_{t_{k}}\left(U, S_{i}\right)}{c}\right)^{2},
$$

where $f$ is the carrier frequency, $c$ is a constant, and $l_{\sigma}$ is $l_{L o S}$ or $l_{N L o S}$ for excessive path losses of LoS and NLoS links respectively.

\section{Problem Formulation}

The data collection maximization issues contains two aspects: $(i)$ the maximization of the number of collected packets $\left(N_{p}\right)$, and (ii) the maximization of the number of nodes that successfully send a packet to the UAV ( $\left.N_{\text {node }}\right)$. Hence, in this paper, we will jointly optimize the two objectives through proposed mechanisms.

1) Optimization of $N_{p}$ : Let,

$$
\zeta_{i k}=\left\{\begin{array}{cc}
1 & U A V \text { communicate with } S_{i} \text { at } t_{k}, \\
0 & \text { otherwise. }
\end{array}\right.
$$

then,

$$
N_{p}=\frac{1}{P_{s}} \sum_{S_{i}} \sum_{t_{k}} \zeta_{i k} \cdot L_{S_{t_{k}}^{i}} \cdot D_{r} \cdot\left(t_{k}-t_{k-1}\right),
$$

in which, $P_{s}$ is the packet size, $D_{r}$ is the data rate, and $L_{S_{t_{k}}^{i}}$ is given through (2). The optimization of $N_{p}$ is presented as,

$$
\begin{aligned}
\mathscr{P}_{1}: & \max _{S_{i}, t_{k}}\left\{N_{p}\right\}, \\
\text { s.t. } \quad & \sum\left(t_{k}-t_{k-1}\right) \leq T, \quad k \geq 1, \\
& \sum \zeta_{i k} \leq N, \forall k,
\end{aligned}
$$

Constraints (5)-(6) imply that, a UAV only communicate with one node at the same time.

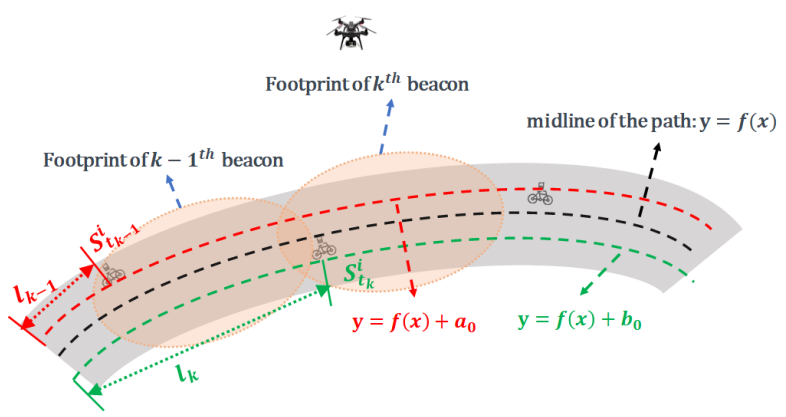

Fig. 3. An illustration of the trajectory of $S_{i}$. In this figure, $l_{k}$ is the curve length.

2) Optimization of $N_{\text {node: }}$ :

$$
\begin{aligned}
\mathscr{P}_{2}: & \max _{S_{i}, t_{k}}\left\{N_{\text {node }}\right\}, \\
\text { s.t. } \quad & \sum\left(t_{k}-t_{k-1}\right) \leq T, \quad k \geq 1, \\
& \sum \zeta_{i k} \leq N, \forall k,
\end{aligned}
$$

where $N_{\text {node }} \triangleq H i s t(I)$, and $I$ is a matrix with $I_{i k}=\zeta_{i k} \cdot i$. Both optimization issues of $\mathscr{P}_{1}$ and $\mathscr{P}_{2}$ are NP-hard combinatorial maximization problem [17]: under the given conditions, its objective is to select items which have unique weight and value to maximize the total value.

\section{Proposed Solutions}

In this section, we will first introduce how to quantity evaluate the transmission opportunity of the nodes and then the proposed optimization algorithms based on it.

\section{A. Transmission Opportunity}

As presented in Fig. 3, suppose that, the midline of the predefined path is given by $y=f(x)$. At $t_{k}$, the UAV sends the $m^{t h}$ beacon message to its coverage, and $S_{i}$ receives it and successfully sends the join message to the UAV. The precise location and speed of the $S_{i}$ which are recorded in join message are obtained by the UAV. The location coordinates is denoted by $S_{t_{k}}^{i}\left(x_{t_{k}}^{i}, y_{t_{k}}^{i}, 0\right){ }^{1}$. The distance between $S_{i}$ and the midline of the path is denoted by $a_{0}=y_{t_{k}}^{i}-f\left(x_{t_{k}}^{i}\right)$ (as presented in Fig. 3). Without loss of generality, we assume that the $S_{i}$ moves along a line which is given by $y=f(x)+a_{0}$ before the $m+1^{t h}$ beacon coming. This assumption is based on an estimation that the influence brought by the lane change of bicycles is negligible compared to the path length.

Suppose that, the $m+1^{t h}$ beacon is sent at $t_{l}$ (then, the $I B D$ $\left.=t_{l}-t_{k}\right)$. Then, the coordinates of $S_{i}$ at $t_{j}\left(t_{k} \leq t_{j}<t_{l}\right)$ is given by,

$$
\left\{\begin{array}{l}
v_{i} t_{j} \doteq \int_{0}^{x_{t_{j}}^{i}} \sqrt{\left(1+\left(y^{\prime}\right)^{2}\right)} d x \\
y_{t_{j}}^{i}=f\left(x_{t_{j}}^{i}\right)+a_{0}
\end{array}\right.
$$

\footnotetext{
${ }^{1}$ In this paper, we consider the vehicles mobile on a flatland and assume that its altitude changes are negligible.
} 


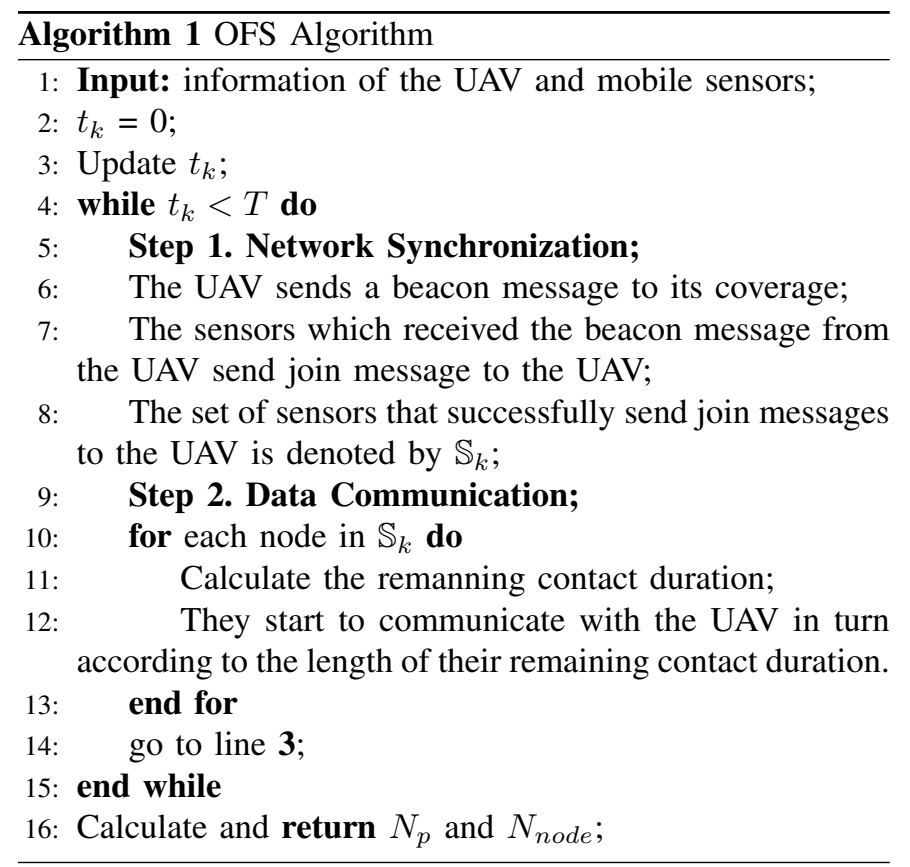

where $\int_{0}^{x_{t_{j}}^{i}} \sqrt{\left(1+\left(y^{\prime}\right)^{2}\right)} d x$ is the curve length of $y=f(x)+$ $a_{0}$ when $x \in\left[0, x_{t_{j}}^{i}\right]$. Accordingly, the coordinates of the UAV at $t_{j}$ can be obtained through,

$$
\left\{\begin{array}{l}
v t_{j} \doteq \int_{0}^{x_{t_{j}}^{u}} \sqrt{\left(1+\left(y^{\prime}\right)^{2}\right)} d x, \\
y_{t_{j}}^{u}=f\left(x_{t_{j}}^{i}\right) .
\end{array}\right.
$$

Let $T_{r d, t_{k}}^{i}$ be the remaining contact duration of the $S_{i}$ and the UAV at $t_{k}$. It means that the $S_{i}$ will out of the range of the UAV after $T_{r d, t_{k}}^{i}$, and it can be obtained through,

$$
T_{r d, t_{k}}^{i} \doteq \frac{1}{v_{i}} \int_{x_{t_{k}}^{i}}^{x_{t_{\lambda}}^{i}} \sqrt{\left(1+\left(y^{\prime}\right)^{2}\right)} d x
$$

with a boundary conditions $d_{t_{\lambda}}\left(U_{t_{\lambda}}, S_{t_{\lambda}}\right)=R$ where $t_{\lambda}=$ $t_{k}+T_{r d, t_{k}}^{i}$ and $R$ is the transmission range of the UAV.

In the following, we will introduce an Opportunity-optimal Frame Selection (OFS) algorithm to enhance the transmission opportunities for those nodes that have short remaining contact duration with the UAV.

\section{B. Opportunity-optimal Frame Selection Solution}

The main procedure of OFS algorithm consists two steps (as shown in Algorithm 1): (a) Network synchronization. The UAV sends a beacon message to its coverage to tell it is coming. The sensors that received the beacon message send join message to the UAV. Then, it obtains the set of nodes that successfully send join messages to the UAV. The set is denoted by $\mathbb{S}_{k}$. (b) Data collection. For each sensor in $\mathbb{S}_{k}$, calculate the remaining contact duration between it and the UAV. Then, all sensors in $\mathbb{S}_{k}$ were given a waiting time with a length which is proportional to its remaining contact duration. Then, it starts communications between the sensors in $\mathbb{S}_{k}$ and the UAV. Perform alternatively the two steps till the end of the collecting time.
TABLE I

SIMULATION PARAMETERS

\begin{tabular}{|l|l|}
\hline Parameters & Value \\
\hline Network size $(N)$ & 200 \\
\hline Collecting time $(T)$ & $300 \mathrm{~s}$ \\
\hline Fly height $(h)$ & $15 \mathrm{~m}$ \\
\hline Data rate $\left(D_{r}\right)$ & $250 \mathrm{kbps}$ \\
\hline UAV speed $(v)$ & $10 \mathrm{~ms}^{-1}$ \\
\hline Transmission range $(R)$ & $100 \mathrm{~m}$ \\
\hline Packet size $\left(P_{s}\right)$ & $127 \mathrm{Bytes}$ \\
\hline Data rate $\left(D_{r}\right)$ & $250 \mathrm{kbps}$ \\
\hline Carrier frequency $(f)$ & $2 \mathrm{GHz}$ \\
\hline $\begin{array}{l}\text { Path loss of LoS and NLoS } \\
\left(l_{L o S} \text { and } l_{N L o S}\right)\end{array}$ & $0 \mathrm{~dB}$ and $3 \mathrm{~dB}$ \\
\hline $\begin{array}{l}\text { Environmental constants }(a \\
\text { and } b)\end{array}$ & 9.61 and 0.16 \\
\hline
\end{tabular}

Through the proposed OFS algorithm, the ones that have shorter remaining contact duration which means it has small transmission opportunity were given higher transmission opportunities. Thereby, the data collection of the system was enhanced.

In the following, we implement two categories of simulations: (a) simulations addressed on simulated movement, and (b) simulations addressed on real experiments movement. In both of (a) and (b), we consider a UAV-aided mobile WSN which consists of one UAV, one data center, and multiple mobile sensors.

\section{Numerous Results}

\section{A. Implementations and Parameters}

In the two categories of simulations, the UAV and the data center are deployed at the beginning of the path, and sensors are deployed within 100 meters from the origin point. In (a), the initial speeds of the sensors are generated by the random function with a boundary of $(1,10) \mathrm{ms}^{-1}$. And its changing of speeds during collecting time is obtained through adding a random disturbance on the initial speeds. In (b), we utilize the real experiment movement data in [4] which are conducted on Jia'er Road, which width and length are 5 meters and 1200 meters respectively, in Tongji University. As presented in Fig. 4(a) and Fig. 4(b), we set 4 waypoints along the Jia'er Road. The UAV starts from the original point to waypoint $P_{1}$ to achieve its given speed, then, to $P_{2}, P_{3}$, and $P_{4}$ in turn (Fig. 4(b)).

The UAV equipped with a Pixhawk [14], [16] and controlled by a autopilot system. Therefore, its trajectory and speed are controlled well which can be seen from Fig. 5. The UAV will hover for 2 seconds in the waypoint because of the control in system. Thus, the speed around waypoint is lower than 5 $m s^{-1}$ (e.g., $P_{2}$ and $P_{3}$ in Fig. 5). The simulated parameters are presented in Table I. 


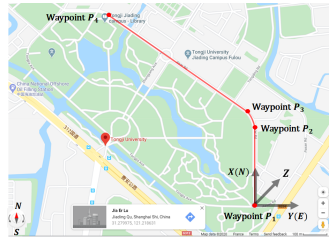

(a) Location map of studied path (b) Flight control system

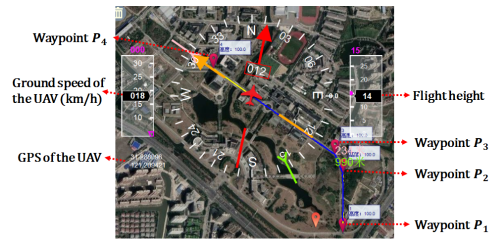

Fig. 4. Presentation of the studied path.

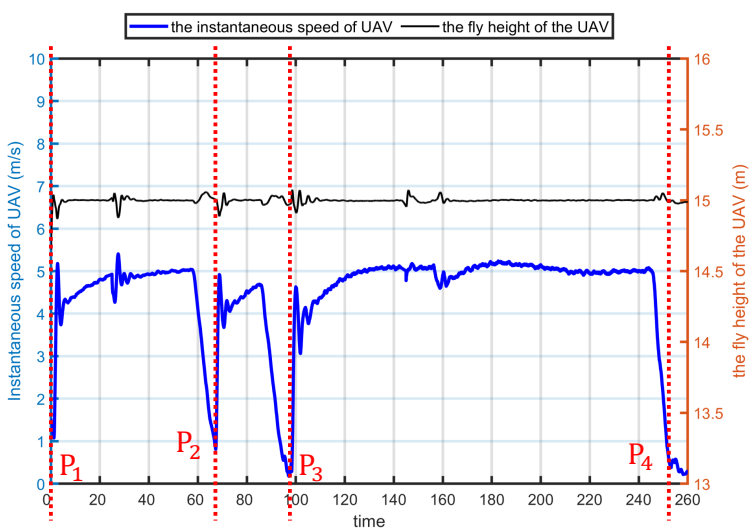

Fig. 5. Presentation of the movement of UAV in real experiment: flying height is 15 meters and the setting speed in control system is $5 \mathrm{~ms}^{-1}$.

\section{B. Results: Simulated Movement}

Fig. 6 presents the data collection performance of the proposed algorithms. The inter beacon duration has impact on both the $N_{p}$ and the $N_{\text {node }}$. The shorter the IBD, the larger the $N_{p}$ and $N_{\text {node }}$. The $N_{p}$ increase as the network size increase if IBD $\leq 50$ ts (10.585 seconds) ${ }^{2}$ and $N \leq 30$ (Fig. 6(a)), and $N_{p}$ starts to leave off when $N>30$. This is because, when the size is too large, there are too much nodes compete to communicate with the UAV which overload of the UAV. It has a little difference on the $N_{\text {node }}$ when IBD $\leq 20 t s$ (that is 4.234 seconds) and $N \leq 50$ (Fig. 6(b). $N>50$, the shorter the IBD, the larger the $N_{\text {node }}$.

Fig. 7 shows the comparison of precise RCD and predicted RCD of four nodes with each has a speed of $\{2,4,6,8\} \mathrm{ms}^{-1}$ when IBD is fixed at $10 \mathrm{ts}$. The fly speed and height of the UAV are $10 \mathrm{~ms}^{-1}$ and $15 \mathrm{~m}$ respectively. The RCD is well predicted through the proposed prediction algorithm because of no environments are considered.

\section{Results: Real Experiments}

Fig. 8 shows the data collection performance of the network using the movement state in real experiment. In Fig. 6, the $N_{p}$ is about 2 times larger than in Fig. 8. This is because the impact of environment in real experiments is much high

\footnotetext{
${ }^{2}$ In this section, the $t s$ is defined as the duration of sending a packet at the lowest data rate (4.8 kbps, see [5] for more details), which is 0.2117 seconds. Then, $50 \mathrm{ts}$ is 10.585 seconds.
}

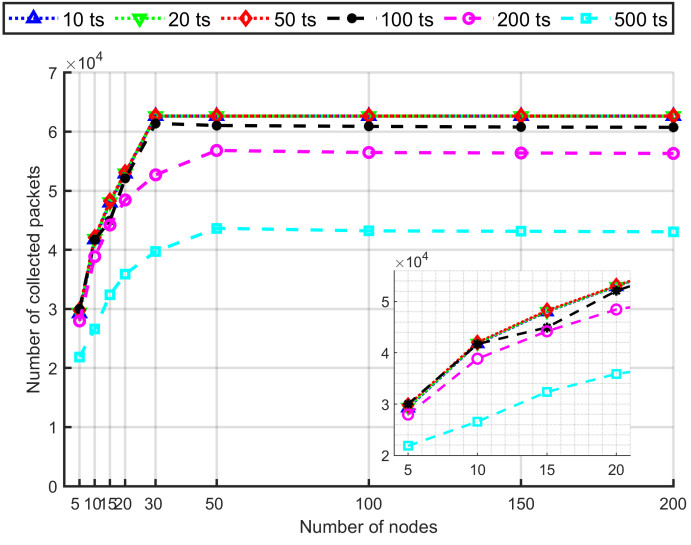

(a) Data collection performance: collected packets, $N_{p}$.

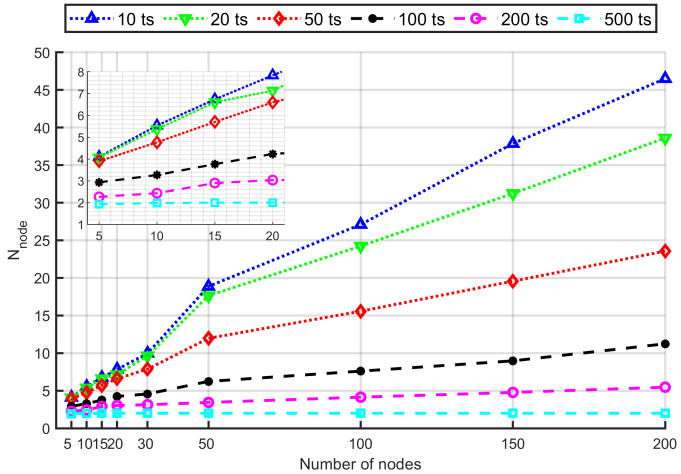

(b) Data collection performance: the number of sensors that participate the communications, $N_{\text {node }}$.

Fig. 6. Results: simulated movement. The data collection performance of the proposed algorithms.

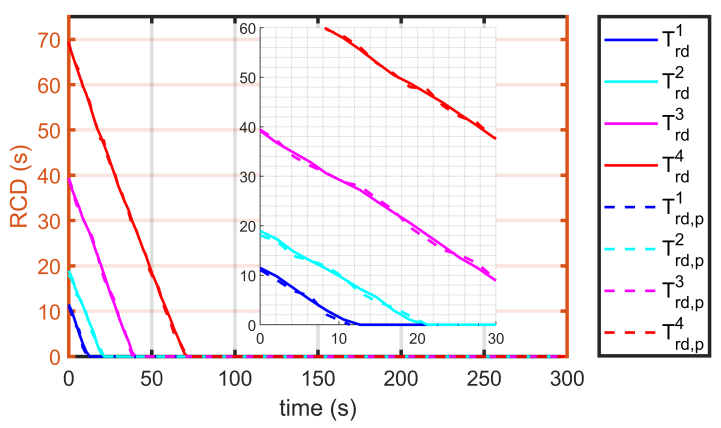

Fig. 7. Results: simulated movement. The comparison between the precise remaining contact duration and the predicted value which is calculated by the proposed mechanism. For clearly comparison, we present four nodes, and fixed the IBD at $10 t s$ (2.117 seconds). In this figure, $T_{r d}$ is the precise value, and $T_{r d, p}$ is the predicted value.

then in the simulations. Similarly to Fig. 6, the data collection performance in Fig. 8, $N_{p}$ and $N_{\text {node }}$, increase as the network size increase. The larger the number of sensors, the larger the number of nodes that have opportunities to communicate with the UAV. Therefore, the $N_{p}$ were enhanced accordingly. 


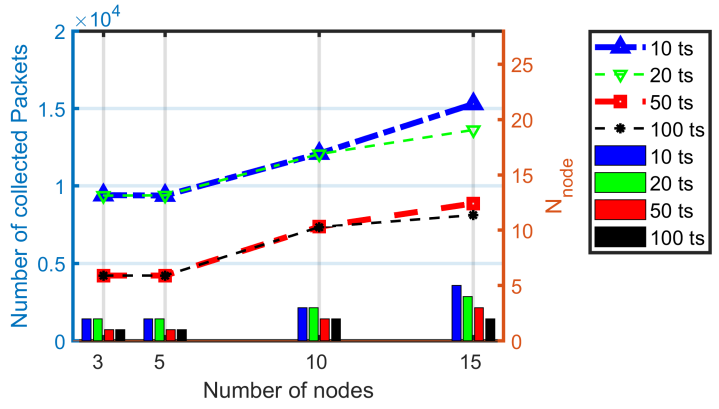

Fig. 8. Results: real experiment. The data collection performance of the proposed algorithms.

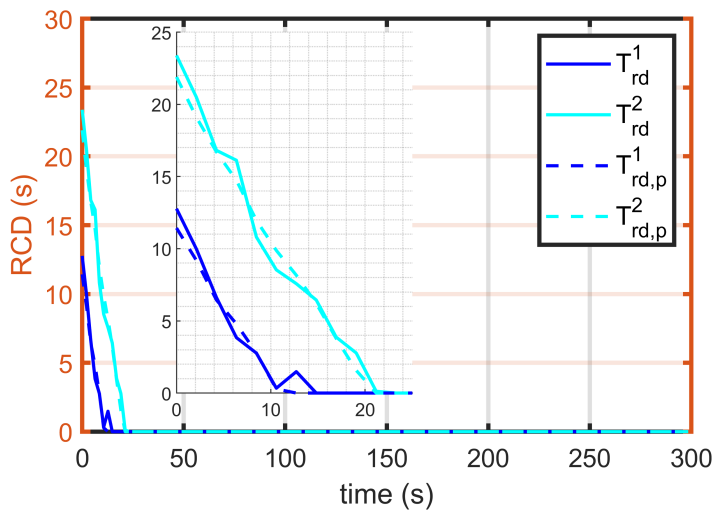

Fig. 9. Results: real experiment. The comparison between the precise RCD and the predicted RCD (using the proposed algorithm in this work) for two nodes (IBD is fixed at $10 \mathrm{ts}, 2.117$ seconds). Here, $S_{1}$ and $S_{2}$ move at 2 $m s^{-1}$ and $4 \mathrm{~ms}^{-1}$ respectively.

It also can be seen that the $N_{\text {node }}$ has very little difference between different IBD. This is because, there are only 15 (maximum) sensors are studied in real experiments. Therefore, the $N_{\text {node }}$ is mainly depend on the initial deployment of the sensors. The predicted remaining contact duration in Fig. 9 has larger deviation than in Fig. 7 because that the movement of the node (including speed value and direction) has more randomness than the movement in Fig. 7.

\section{CONCLUSION}

In this work, we studied the dynamic parameters which are brought by the movement of the UAV and the sensors in the UAV-aided mobile sensor network. Based on these dynamic parameters, we introduce the contact duration between the UAV and the sensors, and propose the prediction mechanism to predict the it. Then, we propose an opportunity-optimal frame selection algorithm, named as OFS algorithm, to enhance the transmission opportunities of those nodes that have very short contact duration with the UAV. Thereby, the data collection performance in terms of the number of collected packets and the number of nodes that successfully participate the communications are improved. Through extensive simulations based on simulated movement and real experiments, we compared the predicted contact duration with the precise value, and examined the effectiveness of the proposed data collection mechanism under different configurations. The results present that the proposed algorithms perform well on system data collection in mobile context. In the future, we aim to propose a framework using multiple UAVs for data collection in mobile sensor networks.

\section{REFERENCES}

[1] M. Mozaffari, W. Saad, M. Bennis, Y. Nam, and M. Debbah. "A Tutorial on UAVs for Wireless Networks: Applications, Challenges, and Open Problems", IEEE Communications Surveys Tutorials, 21, pp. 23342360, 2019.

[2] M. Samir, S. Sharafeddine, C. M. Assi, T. M. Nguyen, and A. Ghrayeb. "UAV Trajectory Planning for Data Collection from Time-Constrained IoT Devices", IEEE Transactions on Wireless Communications, 19(1), pp. 34-46, Jan. 2020.

[3] C. Shen, T. Chang, J. Gong, Y. Zeng, and R. Zhang, "Multi-UAV Interference Coordination via Joint Trajectory and Power Control", IEEE Transactions on Signal Processing, vol. 68, pp. 843-858, 2020.

[4] X. Ma, T. Liu, S. Liu, R. Kacimi, and R. Dhaou. "Priority-Based Data Collection for UAV-Aided Mobile Sensor Network", Sensors, 20, 3034, 2020.

[5] X. Ma. "Data Collection of Mobile Sensor Networks by Drones", [Online Available]: http://docplayer.fr/18339015-These-envue-de-l-obtention-du-doctorat-de-l-universite-detoulouse.html, 2017.

[6] X. Ma, R. Kacimi, R. Dhaou. "Fairness-aware UAV-assisted data collection in mobile wireless sensor networks", in 2016 International Wireless Communications and Mobile Computing Conference (IWCMC), Paphos, Cyprus, pp. 995-1001, 2016.

[7] C. Zhan and H. Lai. "Energy Minimization in Internet-of-Things System Based on Rotary-Wing UAV", IEEE Wireless Communications Letters, 8, pp. 1341-1344, 2019.

[8] Q. Hu, Y. Cai, G. Yu, Z. Qin, M. Zhao, and G.Y. Li. "Joint Offloading and Trajectory Design for UAV-Enabled Mobile Edge Computing Systems", IEEE Internet of Things Journal, 6, pp. 1879-1892, 2019.

[9] C. You and R. Zhang. "3D Trajectory Optimization in Rician Fading for UAV-Enabled Data Harvesting", IEEE Transactions on Wireless Communications, 18, pp. 3192-3207, 2019.

[10] Y. Hu, X. Yuan, J. Xu, and A. Schmeink. "Optimal 1D Trajectory Design for UAV-Enabled Multiuser Wireless Power Transfer", IEEE Transactions on Communications, 67, pp. 5674-5688, 2019.

[11] J. Li, H. Zhao, H. Wang, F. Gu, J. Wei, H. Yin, and B. Ren. "Joint Optimization on Trajectory, Altitude, Velocity, and Link Scheduling for Minimum Mission Time in UAV-Aided Data Collection", IEEE Internet of Things Journal, 7, pp. 1464-1475, 2020.

[12] X. Ma, S. Chisiu, R. Kacimi, and R. Dhaou. "Opportunistic communications in WSN using UAV”, 2017 IEEE Annual Consumer Communications and Networking Conference (CCNC), Las Vegas, NV, pp. 510-515, 2017.

[13] D. Yang, Q. Wu, Y. Zeng and R. Zhang, 'Energy Tradeoff in Groundto-UAV Communication via Trajectory Design", IEEE Transactions on Vehicular Technology, 67(7), pp. 6721-6726, 2018.

[14] PIXHAWK [online]: https://pixhawk.org/

[15] A. Hourani, S. Kandeepan, and A. Jamalipour, "Modeling air-to-ground path loss for low altitude platforms in urban environments", in Proc. IEEE Global Communications Conference (GLOBECOM), Austin, TX, USA, Dec. 2014.

[16] L. Meier, P. Tanskanen, L. Heng, G.H. Lee, F. Fraundorfer, and M. Pollefeys. "Pixhawk: a micro aerial vehicle design for autonomous flight using onboard computer vision", Autonomous Robots, 33(1-2), 21-39, 2012.

[17] Y. Liu, C. Gao, Z. Zhang, Y. Lu, S. Chen, M. Liang, and L. Tao. "Solving np-hard problems with physarum-based ant colony system", IEEE/ACM Transactions on Computational Biology and Bioinformatics, 2017. 